\title{
LA PARTICIPACIÓN SOCIAL ENTRE EL TRABAJO COLECTIVO Y LA BENEFICENCIA PRIVADA: UN ANÁLISIS CON FOCO EN EL CUIDADO INFANTIL $Y$ LAS TRANSFORMACIONES URBANAS EN BARRIOS DEL Gran Buenos Aires, Argentina
}

Laura Santillán Instituto de Ciencias Antropológicas, Facultad de Filosofía y Letras, Universidad de Buenos, Buenos Aires, Argentina Consejo Nacional de Investigaciones Científicas y Técnicas,

Buenos Aires, Argentina laursantillan@mail.com

Resumen: En este artículo, analizamos un conjunto de iniciativas destinadas al cuidado infantil que se organizan con base en el trabajo comunitario en barrios populares de Buenos Aires. La intención es profundizar en los sentidos que asume la participación social en contextos que sobresalen por los cambios urbanos y la desigualdad social.

Palabras clave: Trabajo comunitario, cuidado infantil, participación social, desigualdad, contextos urbanos.

Abstract: In this article we analyze a set of initiatives related to child care. The organization of these initiatives is based on community work in popular neighborhoods of Buenos Aires. The intention is to study in depth the meanings involved in social participation in contexts marked by urban change and social inequality.

Keywords: Community work, child care, social participation, inequality, urban contexts.

Recibido: 12-07-2013 / Aceptado: 24-11-2013 http://revistas.ucr.ac.cr/index.php/antropologia 


\section{Introducción}

En barrios y asentamientos populares de la conurbación Metropolitana de Buenos Aires, las prácticas relativas a la crianza y la educación de la infancia exceden ampliamente las fronteras de las familias de los chicos. En buena medida, los niños y las niñas reciben diversas acciones relacionadas con su cuidado (alimentación, educación y esparcimiento) en virtud de lazos ampliados de parentesco y también en referencia a múltiples intervenciones sociales. Centros para la primera infancia, para la atención de la nutrición y estimulación temprana, así como jardines maternales comunitarios, merenderos ${ }^{1}$ y apoyos escolares ${ }^{2}$ son sólo algunas expresiones de la presencia -en los barrios- de iniciativas destinadas al cuidado infantil. Estas manifestaciones, a cargo de voluntarios externos al barrio y/o los mismos pobladores, responden a una heterogénea y diversificada trama de intervenciones e inserciones en los asentamientos populares. Una serie de acontecimientos -ligados con cambios sociourbanos en nuestra región, en la gestión de las políticas estatales, en los procesos de movilización dentro del campo popular y por parte de las organizaciones de la sociedad civil- promovieron la emergencia de una multiplicidad de asociaciones destinadas a la infancia en distintos enclaves y con acciones que muestran importantes mixturas respecto a los actores y los dominios de la vida social que engloban. Muchas de estas iniciativas - del estado o fuera de él- reactualizan de continuo una forma de trabajo que se define como sociocomunitario.

En esta presentación, nos interesa analizar los modos en que -en asentamientos en donde estamos trabajando- las intervenciones destinadas a los niños y las niñas pertenecientes a los sectores subalternos se organizan estrechamente con base en propuestas referenciadas en el trabajo comunitario y la participación social, en donde convergen heterogéneas y diversificadas influencias, entre ellas la proveniente a los

\footnotetext{
${ }^{1}$ Se llaman merenderos a los espacios comunitarios que abren organizaciones territoriales o vecinos, en donde se ofrece a los chicos, a la salida del horario escolar, una copa de leche.

${ }^{2}$ En referencia a la ayuda que realizan colectivamente organizaciones comunitarias y grupos de vecinos a los niños y las niñas de los barrios populares para completar la tarea que solicita la escuela, juntándolos en domicilios particulares y/o edificaciones de las organizaciones.
} 
condominios cerrados apostados en el lugar. En función de esta diversidad, es nuestra intención discutir una serie de naturalizaciones respecto a la participación social. Entre otros, nos referimos a la tendencia a pensar la participación social como acontecimiento concretado en relaciones horizontales, estructurada por fuera de la conflictividad y las relaciones de poder. Como desarrollaremos en el artículo, en los contextos en donde investigamos, las formas que asume el trabajo sociocomunitario mucho tiene que ver con procesos sociales y políticos más amplios y también con importantes transformaciones que tuvieron lugar en el espacio urbano, que -en la realidad del área Metropolitana de Buenos Aires- están marcadas por la desigualdad social. El análisis que presentamos es parte de una investigación más amplia, de corte cualitativo, antropológico y sustentado en el enfoque etnográfico que venimos realizando desde el 2008. En relación con la indagación empírica, recuperamos el trabajo de campo que efectuamos entre el 2010 y 2012 en un conjunto de asentamientos ubicados en la Zona Norte del Gran Buenos Aires, Argentina. La indagación empírica combinó entrevistas en profundidad y basadas en el enfoque biográfico (Berteaux, 1988) con observación participante. El análisis que presentamos se basa en el registro que desarrollamos de distintos eventos de la cotidianeidad que tienen lugar en los barrios. Entre estos eventos nos referimos a las acciones que se llevan adelante en: espacios dedicados al complemento alimentario ("comedores" y "merenderos"), espacios de "apoyo escolar", instituciones barriales a cargo de fundaciones y los propios pobladores, y las redes barriales organizadas por el Municipio. 


\section{La participación social y el trabajo comunitario en iniciativas destinadas a la infancia}

En nuestro país, Argentina, las iniciativas sociales y comunitarias sobre la atención y el cuidado de los niños y las niñas tienen como marco general las definiciones más estructurales de un conjunto de políticas y programas, formulado principalmente desde el Estado ${ }^{3}$, pero también fuera de él. Nos referimos, por ejemplo, a los organismos internacionales como la Unesco ${ }^{4}$ y Unicef ${ }^{5}$ desde donde se formulan propuestas de intervención y también supervisión sobre los estados y gobiernos nacionales. Desde estas orientaciones más estructurales, es posible advertir algunos aspectos nodales: uno es el interés en promover el desarrollo de los niños y las niñas, como sujetos de derecho y partícipes activos de un proceso de formación integral. Otro aspecto se vincula con la explícita enunciación que se hace de la necesidad de llevar adelante las iniciativas desde el trabajo territorial y sociocomunitario, tendientes a la participación y promoción social de las familias y las comunidades de origen de los niños y las niñas.

Es importante decir que en nuestra región el discurso de la participación comunitaria tiene larga data. Como señalan otros autores, desde, al menos la década del 60 organismos internacionales (como la Cepal y ONU), se han encargado de presentar a la participación social como una actividad "necesaria" en América Latina

\footnotetext{
${ }^{3}$ En relación con las iniciativas gubernamentales en la Argentina, las intervenciones ligadas a la atención y el cuidado de la infancia se exponen en programas específicos que formulan las carteras ministeriales de Educación, Salud y Desarrollo Social. Las líneas programáticas propuestas desde el Estado se referencian principalmente en la ley 26.061 de Protección Integral de los Derechos de Niños, Niñas y Adolecentes sancionada en el 2005 y en la Convención Internacional de los Derechos del Niño de1989, que se constituye en una matriz nodal para la fundamentación de las acciones.

${ }^{4}$ Desde su oficialización en 1946, la Unesco se presenta como uno de los organismos especializados de las Naciones Unidas. Nuestro país, Argentina, es Estado miembro de la Unesco desde 1948, dos años después de su constitución oficial. Como Estado miembro (o como miembro asociado), los países tienen derecho a percibir las ayuda y colaboraciones que se desarrollen en el seno de la Unesco, a la vez que el deber de someter a la Organización, en el momento y la forma que decida la Conferencia General, informes sobre las leyes, reglamentos y estadísticas relativos a sus instituciones y actividades educativas, científicas y culturales, así como sobre el curso dado a las recomendaciones y convenciones que se sugirieran.

${ }^{5}$ Unicef, definido como el Fondo de las Naciones Unidas para la Infancia, desarrolla su labor en más de 190 países y territorios por medio de programas y comités nacionales.
} 
para lograr determinados objetivos en campos sociales específicos y estratégicos como es el de la salud, la educación y la economía (Menéndez y Spinelli, 2006). Con la entrada al nuevo milenio, las sugerencias de los organismos multilaterales de invertir en la capacidad organizativa de los pobres (Banco Mundial, 2000), con atención a los recursos morales y culturales comunitarios de las poblaciones (BID, 2001), y las relaciones informales de confianza y cooperación de la familia y el vecindario (Cepal, 2008) cobraron -sobre todo a través de conceptos tales como capital social- una fuerte difusión e impregnaron en los imaginarios del sentido común científico y en los contextos de implementación de las políticas. Se trata de una formulación que se presenta -entre las distintas intervenciones- con importantes continuidades aun cuando las matrices que las estructuren sean muy diferentes.

Ahora bien, en el caso del Área Metropolitana de Buenos Aires -como en muchos otros enclaves de nuestro país- distintos movimientos y procesos sociales y políticos, han producido que "el barrio" se constituya en el ámbito central -aunque no por ello exclusivo- en donde transcurren las intervenciones sobre el cuidado y la atención de los chicos. Como expusimos en trabajos previos, el escenario local de los barrios -ya superada la década de los 90- continúa siendo sitio en donde se materializan y disputan buena parte de las políticas y programas sociales destinados a los sectores subalternos, entre ellos los centrados en el cuidado y la educación de la infancia (Santillán, 2011, 2012).

Entonces, más allá de que "el trabajo comunitario" sea un objetivo expuesto en las propuestas estatales y en los organismos no gubernamentales, en los contextos barriales acontecen de por sí -en relación con estas intervenciones y también con relativa autonomía a éstas- variadas y diversificadas iniciativas tramadas "en el territorio". En virtud de la modalidad territorializada que asumen estas acciones, los 
lazos de proximidad y asociatividad signan a buena parte -por no decir a todas- de las interacciones y las prácticas puestas en juego. Sin embargo, atentos a no tomar como dadas -una vez para siempre- las relaciones de asociatividad locales -y mucho menos el logro de la participación comunitaria- nos interrogamos varias cuestiones ¿Cómo se producen en escenarios específicos los procesos de participación social? ¿Entre quienes? ¿Qué sentidos asumen los postulados de promoción social en las iniciativas pensadas para los chicos?

\section{La crianza y el cuidado infantil en escenarios urbanos atravesados por intervenciones "multideterminadas"}

Nuestra investigación se sitúa en la Zona Norte del Gran Buenos Aires. Un rasgo que caracteriza a esta zona es la prominente concentración de sectores poblacionales con altos niveles de ingresos monetarios. Esta tendencia acompañó las distintas etapas de expansión del Área Metropolitana bonaerense ${ }^{6}$, de hecho, durante el período denominado de "Organización Nacional", a fines del siglo XIX, esta zona sobresalió por su "distinción"7. La suburbanización metropolitana de Buenos Aires, como señalan otros autores, no contrarrestó, sino que más bien consolidó la diferenciación histórica en nuestro país; es decir, aquella en la cual la zona norte quedara tendencialmente ocupada por sectores sociales de mayores recursos, mientras el Sur-Oeste se constituyese, preferentemente, en el lugar de asentamiento de las clases populares (Pirez, 2004). Sin embargo, en nuestro país, los procesos de suburbanización se concretan bajo formas más complejas y contemplan otras diferenciaciones, además de la marcada por el polo Norte-Sur. Como muchas grandes aglomeraciones urbanas, Buenos Aires experimentó un importante proceso de expansión hacia la periferia

\footnotetext{
${ }^{6}$ El Área o Región Metropolitana de Buenos Aires la conforman la Ciudad Autónoma de Buenos Aires y los Partidos del Gran Buenos Aires que, según desarrollaremos, su extensión tuvo lugar en distintas coyunturas políticas y económicas del país.

${ }^{7}$ Numerosas familias con alto poder adquisitivo poseían residencias permanentes o casonas de veraneo en chacras, por lo común ubicadas sobre las barrancas que dan al río de La Plata.
} 
durante el período comprendido entre 1940 y 1960 (Torres, 2001). Siguiendo la tendencia de otros países de Latinoamérica, este proceso de suburbanización se apartó del patrón "clásico" -por ejemplo de Estados Unidos y Europa- en el cual los sectores de altos recursos se ubicaron, con infraestructura adecuada, en la periferia.

La ocupación del cordón cercano a la ciudad capital y luego de otro más periférico, tiene como protagonistas a los sectores populares. El primer anillo que rodea a la ciudad capital se forma con la instalación de amplios sectores de trabajadores urbanos durante la fase sustitutiva de importaciones entre 1930 y $1940^{8}$. Más adelante, durante las décadas del 50 y 60, a partir de cambios en el patrón industrial y también por el desarrollo del transporte automotor, la población se expandió hacia la segunda corona del conurbano, a través de la compra de terrenos a precios populares, aunque con escasa o nula infraestructura, consolidando así la presencia de barrios precarios y autoconstruidos con múltiples necesidades (Torres, 2001).

El barrio Arroyo $1^{9}$, en donde situamos el análisis forma parte de este último cordón urbano. Su distrito de cabecera, Tigre $^{10}$, presenta profundos contrastes en las condiciones de vida de sus pobladores. En las últimas décadas, las diferenciaciones históricas se profundizaron con el desarrollo de las nuevas suburbanizaciones de las elites. Se acentuaron con el asentamiento de población con altos ingresos en los barrios cerrados durante los años 90, sobre todo en cercanías de las autopistas ${ }^{11}$ (Torres, 2001). Por cuanto en esta área Metropolitana, los asentamientos y las villas de

\footnotetext{
${ }^{8}$ Originariamente, el crecimiento de la conurbación de Buenos Aires tuvo lugar a causa de la extensión de los sistemas ferroviarios. En concordancia con la experiencia de otras partes del país, las localidades del Gran Buenos Aires nacieron a la vera de las estaciones del ferrocarril.

${ }^{9}$ Este barrio, ubicado en el partido de Tigre, tiene una extensión aproximada de 150 manzanas y está habitado por 25.000 personas.

${ }^{10}$ El partido de Tigre conforma uno de los 24 partidos del Gran Buenos Aires y se encuentra ubicado a 30 $\mathrm{km}$ de la Ciudad de Buenos Aires. Ocupa una superficie de $148 \mathrm{~km}^{2}$ de sector continental y $220 \mathrm{~km}^{2}$ de sector de islas. Según el censo poblacional realizado en nuestro país en el 2010, su población es de 380.700 habitantes.
}

${ }^{11}$ Si bien la existencia de barrios cerrados en esta zona del Gran Buenos Aires se retrotrae a los años 70, la década del 90 se torna paradigmática para advertir los niveles de crecimiento y expansión de esta forma de urbanización. 
emergencia, que fueron creciendo en número y extensión, se "entremezclan" en un mismo territorio con lujosas mansiones y parques de barrios y clubes privados. Por ese motivo, esta zona del conurbano resulta un claro exponente de los procesos de polarización social $^{12}$ (Torres, 2001) y/o microfragmentación ${ }^{13}$ (Pírez, 2004) que vienen teniendo lugar desde hace mucho tiempo, pero que sin dudas se profundizaron a partir de la implementación de políticas de corte neoliberal llevadas adelante en los años 90 en nuestro país.

En el barrio Arroyo I, ubicado en esta particular configuración de la Zona Norte, un entramado denso de actores e iniciativas destinadas a resolver diversos problemas a los pobladores (vivienda, trabajo, salud, educación) son parte del paisaje local. En este asentamiento, conviven diversas experiencias comunitarias que tienen como foco el trabajo con los niños. Centros culturales, casas de atención integral de la niñez, centros de educación complementaria, centros dedicados a la nutrición y jardines comunitarios son ejemplos de las acciones que se llevan adelante. En un número importante, estas iniciativas tienen como impulso el accionar del Estado, sobre todo a partir de las propuestas que se ponen en juego a través de programas gubernamentales. En muchos otros casos, las acciones se crearon al calor de los procesos de intervención de organizaciones de base territorial, a partir del trabajo que -con distinta antigüedad en el tiempo- llevan adelante diversos actores sociales: jóvenes militantes, voluntarios externos a los barrios, cuadros de base de la Iglesia Católica y pobladores de los asentamientos.

\footnotetext{
${ }^{12}$ Se trata de una "polarización" urbana y social manifiesta en las transformaciones sufridas en la estructura socioterritorial Metropolitana, pero que en partidos como Tigre los gobiernos locales alentaron a través de políticas de marketing urbano, dejando de lado las funciones de regulación que les compete (Torres, 2001).

${ }^{13}$ Con este término, el autor alude a cómo la instalación de los condominios cerrados en cercanías a los barrios populares preexistentes produjo renovadas modalidades de fragmentación que implica la existencia y configuración -en un espacio socioterritorial muy acotado- de grandes distancias sociales y económicas (Pírez, 2004).
} 
En esta historia de la suburbanización de Buenos Aires -y la creación de muchas instituciones comunitarias- no podemos pasar por alto la conformación y organización colectiva de los asentamientos por parte de los pobladores. En concreto, nos referimos a las ocupaciones de tierras públicas y privadas, llevadas a cabo desde los años 80 , a través de acciones planificadas o espontáneas por parte de pobladores subalternos (Cravino, 1999). Estas tomas -como nuevas formas de hábitat que tienen lugar a partir de la ocupación ilegal, aunque no ilegítima de tierras- así como ponen de manifiesto la desarticulación que muchos hombres y mujeres ya experimentaban como clase proletaria tras el último gobierno de facto en nuestro país, también son elocuentes para reconocer la capacidad de estos mismos sujetos de llevar adelante formas de acción colectiva. Las tomas no sólo incluyeron modalidades de resistencia frente a la represión de las fuerzas de seguridad, sino también distintos niveles de participación de los pobladores, estrategias asociativas para la obtención de servicios e instancias colectivas para la reivindicación de la tierra (Cravino, 1999; Manzano, 2008; Santillán, 2012).

Entre los distintos centros en donde llevamos adelante la indagación, nos detendremos en uno de ellos, al que llamaremos La Morada. Este Centro es paradigmático de una forma de intervención a cargo -casi exclusivamente- de pobladores del barrio. Esta institución, en donde los chicos y las chicas reciben ayuda para las tareas de la escuela, también una copa de leche y talleres de arte, se ubica en un sector interno del barrio Arroyo $\mathrm{I}^{14}$. En esta parte del barrio, las calles dejan de ser de asfalto. Las casas, en su mayoría bajas, van sorteando los arroyos y afluentes de agua que casi sin excepción se encuentran contaminados por los efectos de las industrias apostadas desde hace un largo tiempo en el lugar. Aquí, el tránsito de los pobladores mucho tiene que ver con ir satisfaciendo cuestiones básicas relativas a la reproducción cotidiana (las comidas del día, el traslado de los chicos a la escuela, el cumplimiento de la contraprestación del subsidio que se reciben o la jornada laboral).

\footnotetext{
${ }^{14} \mathrm{El}$ centro La Morada recibe aproximadamente 220 chicos y chicas entre 3 y 12 años en turnos rotativos (entre 9 y $12 \mathrm{~h}$ de la mañana y 16 y $18 \mathrm{~h}$ por la tarde) que se distribuyen en distintas dependencias para desarrollar sus actividades.
} 
Como los establecimientos comerciales se reducen a almacenes y quioscos que algunos pobladores habilitan en pequeños sectores de sus casas, es posible que en determinados domicilios se junten al menos un par de vecinos para la compra de productos. Lo mismo sucede en aquellos hogares en donde tiene lugar la distribución de recursos de los planes sociales (como es en el caso del Plan Más Vida ${ }^{15}$ ) y también en donde los vecinos acceden por préstamo a herramientas $y / 0$ espacios para las actividades de "cuentapropismo" (el acceso a los carros para realizar recolección de material reciclable y la posibilidad del acopio ${ }^{16}$ de este). A la vez, en esta zona del asentamiento, la circulación de chicos y pobladores se produce en algunas casas más. Se trata de domicilios particulares -o que lo eran- pero que ahora se vinculan con acciones destinadas a los niños. Uno de estos domicilios es el del Centro La Morada.

Para llegar a este Centro, la mayoría de los chicos y familiares deben bordear al menos uno o dos de los paredones que "protegen" a uno de los barrios privados que circundan al asentamiento. Recorrer el camino, bordeando para ello los extensos paredones, significa toparse con los elementos de seguridad (cámaras y garitas) que este tipo de emprendimientos privados y suntuosos usa, aunque también con las pintadas que dejan distintas agrupaciones en reclamo de un barrio "sin muros". ${ }^{17}$

\footnotetext{
${ }^{15}$ En alusión al plan estatal que pertenece al Ministerio de Desarrollo Social de la Provincia de Buenos Aires y consiste en la distribución de alimentos y dinero -a través de una tarjeta para sucompra- a mujeres embarazadas y/ a niños y niñas menores de 6 años que pertenecen a familias en situación de vulnerabilidad social.

${ }^{16}$ En referencia con el almacenamiento y separación detallada de los materiales recolectados en la vía pública y que son potencialmente reciclables y se comercializarán.

17 En el asentamiento conviven numerosas organizaciones sociales y políticas que suelen dejar plasmadas en las paredes de los barrios cerrados distintas consignas. Entre ellas puede leerse: "Barrios sin muros", "Liberen a los chetos" [millonarios],algunas de las frases más recurrentes que se dejan como pintadas a lo largo de las instalaciones de los tres barrios cerrados que limitan con el asentamiento Arroyo I.
} 
El Centro La Morada se abrió mientras estaban latentes los episodios de diciembre del $2001^{18}$. Corrían los primeros días del verano del 2002, cuando una vecina del asentamiento, Marta, junto con sus compañeras, las manzaneras ${ }^{19}$ que trabajaban igual que ella en el Plan Más Vida, buscan la forma de paliar los problemas acuciantes de alimentación y otras necesidades que vivían muchos chicos de la zona. Para ello, abrieron un pequeño espacio en la vivienda de Marta y comenzaron a ofrecer la copa de leche. Con el tiempo se fueron sumando otras actividades: entre ellas las clases para la ayuda escolar y la recreación para los más pequeños. En esta experiencia, como ocurre en muchas otras dentro del asentamiento, las iniciativas relativas al cuidado de los chicos involucran de lleno relaciones de proximidad y conocimiento mutuo. En la vida cotidiana del Centro La Morada, un grupo de pobladores se constituye en los cuidadores de los niños. Por cierto, se trata de intervenciones asociadas al cuidado y la educación, que aun cuando se llevan adelante en relaciones basadas en la confianza y con una importante "informalidad", suceden fuera del hogar del niño y/o la niña e incluyen actividades específicas que se planifican, se ponen en juego y evalúan con importante regularidad. En ese escenario de actuación, el conocimiento local, dado fundamentalmente porque quienes participan en las acciones son parientes $y / o$ vecinos próximos, está muy presente en este tipo de iniciativas de base y otorga una importante cuota de fluidez en las interacciones y decisiones cotidianas. Esto no quita que en un sinfín de circunstancias la horizontalidad

\footnotetext{
${ }^{18}$ Nos referimos al agravamiento progresivo de las medidas económicas y políticas en nuestro país hacia fines del siglo XX, que producen las movilizaciones y estallidos populares de los días 19 y 20 de diciembre del 2001 en donde tiene lugar la destitución del entonces presidente Fernando De la Rúa.

19 El plan estatal Más Vida contempla una amplia red integrada por trabajadoras vecinales (mayoritariamente mujeres) quienes reciben el nombre de manzaneras. Estas tienen a su cargo a las beneficiarias que viven en su misma "manzana"; es decir, la fracción de terrenos que quedan comprendidas entre cuatro calles.
} 
Presente fisuras y quede permeada por situaciones de conflictividad. Entre otros, el hecho de que los cuidadores sean vecinos, parientes y conocidos de los padres de los niños interpela de manera aguda a quienes llevan adelante las iniciativas: en el marco de relaciones profundamente fluidas y estrechas deben demostrar en forma casi permanente su "idoneidad" en relación a las tareas que se proponen ${ }^{20}$.

En experiencias como La Morada, se materializa una forma de trabajo con importante arraigo en lo comunitario y el trabajo en red. Al menos, de esta manera, es reconocida esta iniciativa por parte de los protagonistas de las acciones y también por parte de las distintas instituciones con las cuales la experiencia dialoga: el Estado local, provincial y/o nacional y también diversas organizaciones no gubernamentales. Un ejemplo de ello es la invitación que el municipio le hace al Centro La Morada para que participe de la red barrial, con antigua data en el lugar, pero que el gobierno local se propuso revitalizar. Según los organizadores de dichas reuniones, la invitación se fundamenta a partir "del tiempo" de trabajo que el Centro tiene en el barrio, su experiencia acumulada en el trabajo en red y la realización de las acciones basadas en la participación comunitaria" ${ }^{21}$. Nos adentramos así a una de las dimensiones más fuertemente valoradas en las experiencias territoriales dirigidas a los niños: aquella referida al trabajo comunitario y la participación social.

En el campo académico y el discurso social, las categorías de "trabajo comunitario" y "en red" ayudan por cierto a reconocer rápidamente una modalidad de acción anclada en el "territorio". Sin embargo -según consideramos- muchas veces estas categorías dejan encubierto una serie de cuestiones que nos parecen nodales. No solo nos referimos a quiénes son -en concreto- las personas (individuales y colectivas) que intervienen en esta o aquella iniciativa "comunitaria", sino -y fundamentalmente- sus relaciones y posicionamiento en un marco más amplio de

\footnotetext{
${ }^{20}$ Alcanzar una "buena reputación" forma parte de la mayoría -por no decir de todas- de las acciones sociales y comunitarias dedicadas en nuestra región al cuidado y la atención de los niños. En el caso que traemos, la exigencia de lograr una "buena reputación" involucra de lleno a los pobladores que deciden abrir un espacio destinado al cuidado y la educación de los niños.
}

${ }^{21}$ Referencias extraídas de los registros de campo de las reuniones de "red barrial" que se organiza en el Barrio Arroyo I. 
correlación de fuerzas. Porque, como dijimos, en la región metropolitana bonaerense hay una batería amplia y diversificada de actores sociales con presencia en los barrios populares. En la Zona Norte, cuadros de base de la Iglesia Católica, militantes sociales, referentes barriales, técnicos y operadores de los programas estatales son algunos de los actores que transitan y trabajan cotidianamente en los asentamientos. A la vez, no son los únicos. En barrios como Arroyo I, como en muchos otros de la misma zona, una presencia insoslayable es la vinculada con la beneficencia privada. Dicha presencia se liga -como adelantamos- con la instalación de las urbanizaciones cerradas y también con la difusión de la llamada "responsabilidad social empresarial"22. En ese marco, en función de las tareas cotidianas, centros como La Morada -y también fuera de élentran en contacto con fundaciones y organizaciones del Tercer Sector ${ }^{23}$. Se trata de una intervención ligada a la beneficencia privada que se presenta bajo formas renovadas; entre ellas, se destaca la apelación que realizan al trabajo comunitario y la pretensión del logro de la promoción social de los pobladores, los niños y las familias.

\section{Las fundaciones y la beneficencia privada: los (auto) convidados al trabajo territorial y comunitario en las iniciativas ligadas a la infancia}

Una pregunta que venimos planteando desde el inicio del artículo es ¿Quiénes intervienen, en concreto, en las definiciones sobre la participación y el trabajo comunitario cuando hablamos de las iniciativas de atención a la infancia en los asentamientos populares? En barrios como Arroyo I, hay una presencia cotidiana de

\footnotetext{
${ }^{22}$ Bajo esta denominación, se autodenominan las iniciativas que llevan adelante empresas con fines de lucro, de asistencia social a poblaciones económicamente desfavorecidas.

${ }^{23}$ Se conoce con el nombre de Tercer Sector o Sector Social a la congregación de organizaciones autodefinidas como "no estatales" y "sin fines de lucro", cuya denominación se sustenta en la intención de diferenciarse del Estado y el mercado. Reconociendo una diversidad de trayectorias y objetivos de estas entidades en la Argentina, debe señalarse que este espacio se consolida como campo y toma protagonismo central en los años 90 de la mano de las ya mencionadas reformas en el Estado y de las recomendaciones de los organismos internaciones de crédito y sus nuevas líneas de financiamiento (Rodríguez, Trinchero y Woods, 2001).
} 
organizaciones vinculadas con el tercer sector no del todo visibilizada, al menos para quienes son foráneos del lugar. Recordemos que estos asentamientos se caracterizan porque su población padece condiciones sociales y económicas aùn muy signadas por la desigualdad social. Son condiciones que se manifiestan en un sinfín de dimensiones: en los problemas de infraestructura de las viviendas, en la falta de servicios básicos (como cloacas), en la contaminación de arroyos y afluentes. Estas necesidades, a la vez, conviven con el levantamiento de edificaciones que erigen estas fundaciones. Como anticipamos, en la Zona Norte del Gran Buenos Aires, desde hace un tiempo a esta parte, un conjunto -no muy numeroso, pero influyente- de fundaciones y benefactores particulares llevan adelante una batería diversificada de intervenciones en el lugar ${ }^{24}$. La existencia de estas instituciones se vincula con varias cuestiones, en buena medida se liga con la acción que emprenden los condominios cerrados que circundan los asentamientos; en otros, con la actuación de empresas de reconocido peso en nuestro país ${ }^{25}$. La presencia de este tipo de instituciones se manifiesta, por ejemplo, en el levantamiento de obras de importante envergadura en donde los chicos y chicas del barrio realizan, entre otras, actividades de hockey, arte, ajedrez, apoyo escolar y orquesta musical. Estas obras incluyen también acciones para la primera infancia, vinculadas principalmente con la estimulación temprana y el desarrollo de los más pequeños. En este último caso, las familias de los niños -sobre todo las madresquedan interpeladas de lleno mediante la convocatoria a cursos, reuniones y entrevistas individuales ${ }^{26}$. Por cierto, estas no son las únicas maneras de intervenir de estas

${ }^{24}$ El registro cuantitativo de las fundaciones y los benefactores en la zona va variando debido al gran dinamismo que asumen en el tiempo. En el barrio Arroyo I, en el momento cuando realizamos nuestro trabajo de campo, sobresalía la presencia y continuidad de tres fundaciones. Una de ellas de pertenencia religiosa, con más de una decena de trabajadores y voluntarios. Las dos restantes fundaciones son laicas, una de ellas vinculada con uno de los condominios privados y la otra con voluntarios provenientes de distintos barrios del partido. El personal que trabaja en estas organizaciones es cuantitativamente alto, a la vez muy cambiante. Asimismo, en estas fundaciones hay benefactores que colaboran en eventos y circunstancias puntuales.

${ }^{25}$ En esta presentación nos dedicaremos a las iniciativas llevadas adelante por los barrios cerrados y no a aquellas más estrechamente vinculadas con lo que se conoce como Responsabilidad Social Empresarial.

${ }^{26}$ Cabe decir que la intervención de estos centros se extienden también hacia otras acciones destinadas a los adultos, como aquellas relativas a la oferta de cursos (de camarero, atención al cliente y ventas, 
fundaciones. Es usual que representantes de las mismas visiten de manera regular a las organizaciones de base del barrio -al menos algunas de ellas- y comiencen allí un trabajo que definen como "colaborativo" y "favorecedor" de la resolución de las necesidades del lugar. Desde las presentaciones escritas que estas organizaciones realizan, un tópico sobre el que frecuentemente se vuelve es el de la postulación de un trabajo horizontal, participativo y conducente a la promoción social:

Abrazamos con fuerza nuestra tarea de ampliar las libertades de los vecinos, progresando en comunidad.

Convencidos de que, mediante un proceso de empoderamiento, los vecinos del barrio ampliarán sus libertades, ejercitaran mejor condición de ciudadanos y harán del anhelo de progresar un estímulo permanente para sus vidas y vecinos $^{27}$.

Cabe señalar que desde las asociaciones hay una insistencia en el reconocimiento de las capacidades de los vecinos, aun cuando -al menos desde sus fundamentaciones- pareciera resultar importante reforzar sus competencias.

Tal como dijimos, la presencia de estas fundaciones mucho tiene que ver con los cambios acontecidos en nuestra región en la configuración urbana y las definiciones de las intervenciones del Estado. En nuestro país, desde los años noventa somos testigos de una pronunciada expansión de la periferia de la ciudad metropolitana de Buenos Aires, entre otros, a partir del levantamiento de las llamadas urbanizaciones cerradas,

recepcionista, cocina, panadería, oficial de mantenimiento, jardinería, costura, peluquería, alfabetización digital, operador administrativo contable, club del emprendedor, call center) que se presentan dentro del área de capacitación para el trabajo y que en muchas ocasiones implica luego la contratación de algunos de los participantes en empleos ligados con el rubro de servicio (limpieza, jardinería, servicio del rubro cocina) en los barrios cerrados.

27 Referencias extraídas del material de difusión de las fundaciones. Omitimos intencionalmente el nombre de las fundaciones con el fin de preservar el anonimato de nuestros entrevistados, entre ellos $-y$ principalmente- los pobladores de los barrios populares. 
destinadas exclusivamente a los sectores económicos medios y altos ${ }^{28}$ (Girola, 2007). La instalación de los barrios cerrados -correspondiente a lo que algunos autores definen como "ciclo posexpansivo urbano con trazado excluyente" que comienza en $1976^{29}$ - se produce a partir de un "ordenamiento territorial" y de un uso del suelo (en el contexto de la dictadura militar) que cercenó el crecimiento de los loteos populares de tierra y que continuó en los 90 a través de las orientaciones neoliberales que asumió el Estado y el peso creciente de las inversiones de actores privados internacionales. Es decir, la década de los 90 , en un escenario marcado por la "estabilidad monetaria" -y el papel acondicionador que adoptó el Estado- produjo la expansión de este tipo de residencia urbana en un radio que va desde los 30 a $70 \mathrm{~km}$ del centro de la ciudad de Buenos Aires, en tierras adquiridas a muy bajo costo a partir de su "escasa rentabilidad" y valor en el mercado por situarse en zonas rurales o semirurales, en varios casos con características de inundabilidad (Ríos, 2005). Este es el caso para la Zona Norte, en donde realizamos la investigación y que concentra el mayor porcentaje de ubicación de urbanizaciones cerradas ${ }^{30}$.

En los alrededores del asentamiento Arroyo I, se ubican tres barrios cerrados. La instalación de estos emprendimientos implicó una serie de medidas -con sus correspondientes efectos en los alrededores del predio- tales como la polderización del suelo $^{31}$ y la presentación de unos planes directores, que incluyeron la planificación del manejo de situaciones de desastres. Ello significó la concreción, como efectúan emprendimientos en otras zonas, de una suerte de decálogo por parte de los condominios privados (denominado Evaluación del Impacto Ambiental) que incluyen la explicitación de una batería de acciones frente a posibles contingencias. Algo a

\footnotetext{
${ }^{28}$ Entre otros, las urbanizaciones cerradas se caracterizan por el cerramiento o la privatización de un área de uso exclusivo donde se concentran las viviendas y servicios, cuyo acceso se restringe a los propietarios mediante rigurosos mecanismos de vigilancia (Girola, 2007).

${ }^{29}$ Coincidente con el gobierno de facto que tuvo lugar en nuestro país, Argentina, entre 1976 y 1983.

${ }^{30}$ En la zona norte se ubica el $67 \%$ de los emprendimientos de barrios privados del país (Girola, 2007).

${ }^{31}$ Es decir, el tratamiento del suelo para su no "inundación" que tiene efectos negativos concretos para los asentamientos aledaños; tales como, cambios en el escurrimiento de las aguas en el área, que potencia procesos de inundación y anegamiento de aguas y cambios en la funcionalidad del sistema de humedal en cuanto a la retención de los excedentes hídricos (Ríos, 2005).
} 
destacar -como contribuyen a señalar trabajos que nos anteceden (Ríos, 2005)- es que dentro de las contingencias, los emprendimientos suelen englobar tanto peligros físicos -naturales (inundaciones y tornados) como tecnológicos-sociales (mencionando entre ellos al "vandalismo" y las "revoluciones sociales"). En relación con estos últimos peligros, es que desde los barrios privados se fueron motorizando una serie de acciones directas destinadas hacia los pobladores de los asentamientos; tales como, la creación de centros de capacitación, educación y esparcimiento. Según se desprende del trabajo de campo, son acciones que están fuertemente orientadas hacia la búsqueda de una gramática de pacificación en las relaciones con las poblaciones aledañas. Como veremos seguidamente, se trata de una tendencia hacia la pacificación sustentada y llevada adelante a través de prácticas directas, y también más intersticiales, de regulación y control social sobre los pobladores destinatarios de las acciones (los niños y las familias, como también los vecinos a cargo de las organizaciones barriales).

Las acciones se fundamentan a partir de la construcción de todo un frente discursivo (Fonseca, 2011) referido al "trabajo comunitario" y la "participación" de los pobladores de los barrios aledaños:

Nuestra Misión es mejorar la calidad de vida de los vecinos del Barrio con el compromiso y la participación de los vecinos, las instituciones y los barrios aledaños. Nuestra Visión es ser un modelo de interrelación comunitaria replicable que permita, a partir de los recursos de una comunidad, provocar un cambio profundo y duradero en el mejoramiento de sus poblaciones vecinas. Nuestra Filosofía se sustenta en la convicción que solo podremos cumplir nuestra misión de mejorar la calidad de vida de las personas del Barrio en el intercambio recíproco con el otro. Este intercambio significa trabajar y accionar con y junto al otro, permitiendo el despliegue de las potencialidades de ambos ${ }^{32}$.

\footnotetext{
${ }^{32}$ Extraído del material de folleto de una de las fundaciones que gestiona sus acciones en el barrio Arroyo I, que previamente nombramos ligada a los condominios privados.
} 
En el marco de las iniciativas que realizan fundaciones como éstas, los merenderos y los talleres de apoyo escolar, por ejemplo, descriptos como espacios grupales complementarios a la jornada de la escuela, son referenciados por las asociaciones filantrópicas como una forma muy importante del buscado "desarrollo comunitario".

Ahora bien, en la permanencia en el trabajo de campo, algo que se destaca es que si bien las fundaciones realizan varias de estas acciones en los Centros que han levantado (es el caso de las clases de hockey, ajedrez y arte) muchas otras las llevan adelante con base en el trabajo que ejecutan los propios pobladores. Este es el caso de las experiencias de apoyo escolar y los merenderos, que si bien son anunciadas como parte de las acciones de intervención de las asociaciones sin fines de lucro, en realidad existen y se mantienen a partir del trabajo que cotidianamente llevan adelante grupos de pobladores en instalaciones que les son propias y sostienen ellos mismos con un marcado esfuerzo (en relación a los gastos de luz, material didáctico, cobertura de copa de leche a los niños). Por cierto, las fundaciones realizan una serie de donaciones, en general acotadas, pero que son muy influyente en términos simbólicos: los apoyos escolares que reciben estas donaciones quedan insertos dentro de esta propuesta de “inclusión”, sustentada en retóricas que aluden al trabajo comunitario y la participación social (de la comunidad) y que encierran por cierto una densa trama de obligaciones y distribución desigual de las responsabilidades.

Como dijimos, en centros como La Morada, un grupo de pobladores de los asentamientos son los protagonistas cotidianos de las acciones. En función de este lugar (como educadores y cuidadores de los niños), estos pobladores son invitados con frecuencia por parte de las fundaciones a participar de instancias de capacitación. Se trata de cursos y seminarios que se llevan adelante en los colegios privados ubicados dentro de los condominios cerrados. Cabe señalar que la agenda de estas capacitaciones está organizada por entero por las fundaciones y los particulares que se acercan a los centros comunitarios. Asimismo, los habitantes de los asentamientos que se constituyen en referentes de los merenderos, comedores y talleres para los chicos, son compelidos a participar de reuniones que van sedimentando la idea de una "red" entre los centros comunitarios. Pero en la cotidianeidad de las acciones - de estos 
centros- la realización de un trabajo articulado y la influencia de la/s fundación/es es relativa. Decimos relativa, porque si bien los habitantes de los condominios cerrados circulan por las asociaciones de base, la organización del trabajo cotidiano queda enteramente -en todas las experiencias- a cargo de los pobladores, a la vez son objeto de continuos mecanismos de vigilancia y control. Entro otros -y principalmente- dicho control recae en el uso que los centros hacen de las ayudas materiales recibidas.

Para finalizar, diremos que si bien las vinculaciones entre los referentes barriales y las fundaciones son muy significativas en virtud de la regularidad y la relativa magnitud de los donativos, constituye uno de los puntos de tensión más sensible en la relación con el resto de los habitantes delos asentamientos populares. Es decir, las cadenas de dones y contra dones imprimen a las organizaciones comunitarias que reciben la ayuda un plus de esfuerzo en pos de la necesidad de demostrar -en este caso a los propios vecinos- no tan solo idoneidad en las tareas, sino también el uso honesto de los recursos.

\section{Palabras finales}

El registro en profundidad en el barrio Arroyo I nos aproximó al reconocimiento de iniciativas vinculadas con la atención a la infancia a cargo de actores heterogéneos, con distintos protagonismos y procedencias. Tal como constatamos desde el trabajo etnográfico, junto con las instituciones más formalizadas del Estado (como la escuela y los espacios dedicados a la primera infancia) en las fracciones más internas de los asentamientos y barrios populares de la Zona Norte del Gran Buenos Aires, se levantan múltiples y diversificadas iniciativas destinadas a los chicos y las chicas. Dichas acciones, sustentadas en la mayoría de los casos, en el trabajo comunitario y la participación social nos conducen a sugestivos debates e interrogantes. 
Como expusimos en el trabajo, desde los programas estatales y también en las sugerencias de los organismos internacionales, se insiste en la necesidad de promover el desarrollo de los niños y las niñas pertenecientes a los sectores subalternos, sobre todo a través de iniciativas que promulguen la participación y promoción social. Al respecto, la retórica de la participación y el trabajo sociocomunitario se tornan nodales. En la contemporaneidad no es posible desatender -desde la investigación- toda una producción de lineamientos teóricos y de propuestas de cursos de acción orientados a modular determinadas acciones por parte de las "familias" de los niños y sus comunidades de origen como formas de propiciar el desarrollo de sus procesos educativos/escolares y de efectivizar el cumplimiento de sus derechos. Sin embargo, hay otros actores, distintos a los usualmente reconocidos, que fijan posición y producen sentidos en torno a la "participación comunitaria". Como hemos dado cuenta en la exposición, sería desacertado desprender el trabajo que realizan las experiencias comunitarias de movimientos más globales, vinculados fuertemente con la historicidad de este tipo de iniciativas en los barrios populares y con el tipo de interacciones y lazos que los pobladores estrechan con actores externos. En esa dirección, hemos incluido en el análisis la presencia y el trabajo que llevan adelante las asociaciones ligadas con las fundaciones sin fines de lucro y el tercer sector. Las cuales, sustentándose en una reapropiación particular de las retóricas de la participación en boga, complejizan el escenario y los procesos de actuación de quienes cotidianamente llevan adelante iniciativas en los asentamientos ubicados en las periferias del Área Metropolitana.

Según entendemos, así como no es posible pensar la actuación de los pobladores que producen iniciativas vinculadas con el cuidado y la atención de los niños por fuera de las formas de relación con las agencias del Estado, tampoco es posible soslayar las vinculaciones con los barrios más onerosos que circundan el lugar. Por cierto, de ningún modo son vinculaciones libres de una serie de decisiones que los propios pobladores de los asentamientos asumen, ya sea de distanciamiento, acuerdos y disensos y que se inscriben y aluden a relaciones y procesos más amplios de fragmentación, asimetría y desigualdad social. 


\section{Referencias bibliográficas}

Banco Interamericano de Desarrollo (BID), 2001: Iniciativa interamericana de capital social, ética y desarrollo. Recuperado el 20 de agosto de 2001, de www.iadb.org/etica/inicitiva.cfm.

Banco Mundial, 2000: Poverty. Recuperado el 20 de agosto de 2001, de www.worldbank.org/poverty/scapital/index.htm.

Bertaux, D. (1988). El enfoque biográfico. Su validez metodológica, sus potencialidades. Cuadernos de Ciencias Sociales. Historia Oral e Historia de Vida, 18, 55-80.

Comisión Económica para América Latina y el Caribe (CEPAL), 2008: Futuro de las familias y desafíos de las políticas. Irma Arriaga editora, Santiago de Chile.

Cravino, C. (1999).Los asentamientos del Gran Buenos Aires. Reivindicaciones y contradicciones. En M.R. Neufeld, S. Tiscornia, M. Grimbreg y S. Wallace (eds), Antropología Social y Política. Hegemonía y Poder: el mundo en movimiento (pp. 261-284). Buenos Aires: EUDEBA.

Fonseca, C. (2011). Tecnologías globales de la moralidad materna: políticas de educación para la primera infancia en el Brasil contemporáneo. En I. Cosse, V. Llobet, C. Villalta y M.C. Zapiola (eds), Infancias, políticas y saberes en Argentina y Brasil (Siglo XIX y XX) (pp. 175-204).Buenos Aires: El Teseo.

Girola, F. (2007). El surgimiento de la megaurbanización en la Región Metropolitana de Buenos Aires consideraciones en torno a las nociones de ciudad-fragmento y comunidad purificada. Estudios demográficos urbanos, 22 (2), 363-397. 
Manzano, V. (2008). Etnografía de la gestión colectiva de las políticas estatales en organizaciones de desocupados de la Matanza- Gran Buenos Aires. RUNA, 28, 77-92.

Menéndez, E. y Spinelli, H. (2006). Participación Social. ¿Para qué?.Buenos Aires: Lugar Editorial.

Pírez, P. (2004). La configuración Metropolitana de Buenos Aires: expansión, privatización y fragmentación. Realidad Económica, 208, 111-134.

Rodríguez, K., Trinchero, H. Woods, M. (2001).La Consulta del Banco Mundial a la sociedad civil en la Argentina. Economía política de la participación.Realidad Económica, 180, 91 - 111.

Ríos, D. (2005). Planificación urbana y desastres de inundación: las urbanizaciones cerradas polderizadas en el Municipio de Tigre. Economía, Sociedad y Territorio, $17,63-83$.

Santillán, L. (2011). El cuidado infantil, la vida familiar y las formas en que se territorializan las intervenciones sociales: un estudio en barrios populares del Gran Buenos Aires. En I. Cosse, V. Llobet, C. Villalta y M.C. Zapiola (eds), Infancias, políticas y saberes en Argentina y Brasil (Siglo XIX y XX) (pp. 287310).Buenos Aires: El Teseo.

Santillán, L. (2012). Quiénes educan a los chicos. Infancia, trayectorias educativas y desigualdad. Buenos Aires: Biblos.

Torres, H. (2001). Cambios socioterritoriales en Buenos Aires durante la década de 1990. EURE, 27 (80), 33-56. 